\section{International Scientific Journal Theoretical \& Applied Science}

p-ISSN: 2308-4944 (print) e-ISSN: 2409-0085 (online)

Year: $2018 \quad$ Issue: $06 \quad$ Volume: 62

Published: $25.06 .2018 \quad$ http://T-Science.org
Unona Krahmaleva

Candidate of Science

Taraz State University named M.H.Dulaty

Assylay Bekmurzayeva

Graduate student of the 4-th course of the specialty «Mathematics»

Taraz State University named M.H.Dulaty

SECTION 2. Applied mathematics. Mathematical modeling.

\title{
SOLVING OF $n$-ORDER LINEAR DIFFERENTIAL EQUATIONS WITH CONSTANT COEFFICIENTS IN MAPLE PROGRAM
}

Abstract: In the Article work conducted the research of methods of solving of n-order linear differential equations with constant coefficients of the computer algebra Maple. The automated mathematical programs with application of a mathematical Maple package are developed for finding of the analytical solution of the (homogeneous and in homogeneous) 3 -order linear differential equations with constant coefficients.

Key words: differential equations, problems, Maple.

Language: Russian

Citation: Krahmaleva U, Bekmurzayeva A (2018) SOLVING OF n-ORDER LINEAR DIFFERENTIAL EQUATIONS WITH CONSTANT COEFFICIENTS IN MAPLE PROGRAM. ISJ Theoretical \& Applied Science, 06 (62): 86-91.

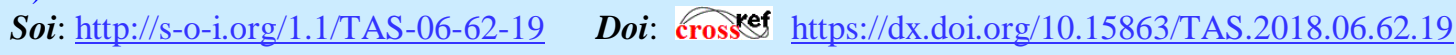

\section{РЕШЕНИЕ ЛИНЕЙНЫХ ДИФФЕРЕНЦИАЛЬНЫХ УРАВНЕНИЙ $n$-ПОРЯДКА С ПОСТОЯННЫМИ КОЭФФИЦИЕНТАМИ В СРЕДЕ МАРLЕ.}

Аннотация: В статье проводится исследование методов нахождения решения линейных дифференциальных уравнений n-го порядка с постоянными коэффициентами средствами компьютерной алгебры Maple. Представлены автоматизированные математические программы с применением математического пакета Марlе для нахождения аналитического решения линейных дифференциальных уравнений (однородных и неоднородных) -го порядка и выше с постоянными коэффициентами.

Ключевье слова: дифференциальное уравнение, однородное линейное уравнение, неоднородное линейное, с постоянными коэффициентами.

\section{Introduction}

В настоящее время, существуют различные подходы к решению дифференциальных уравнений. Первый подход связан с реализацией на бумаге, если известны методы решения уравнений, что представляет собой достаточно трудоемкий процесс. Компьютерная реализация алгоритма на каком - либо языке программирования является вторым подходом. Третий, основан на применении имеющихся современных систем компьютерной математики. В этих системах имеются процедуры реализации необходимых алгоритмов решения. Именно этот подход является наиболее рациональным не только с точки зрения избегания ошибок, но и сокращения времени решения. Этот подход предполагает не только знание алгоритмов решения, но и ихх особенностей, для правильного использования достоинств и недостатков, а так же интерпретации результатов.

\section{Materials and Methods}

Рассмотрим задачу нахождения аналитического решения линейных однородных дифференциальных уравнений $n$-го порядка с постоянными коэффициентами:

$$
a_{0} y^{(n)}+a_{1} y^{(n-1)}+a_{2} y^{(n-2)}+\ldots+a_{n-1} y^{\prime}+a_{n} y=0
$$




\begin{tabular}{l|lr|ll|ll} 
& ISRA (India) & $=\mathbf{1 . 3 4 4}$ & IS $($ USA) & $=\mathbf{0 . 9 1 2}$ & ICV (Poland) & $=\mathbf{6 . 6 3 0}$ \\
Impact Factor: & ISI (Dubai, UAE) & $=\mathbf{0 . 8 2 9}$ & PUHL (Russia) & $=\mathbf{0 . 2 0 7}$ & PIF (India) & $=\mathbf{1 . 9 4 0}$ \\
& GIIF (Australia) & $=\mathbf{0 . 5 6 4}$ & ESJI (KZ) & $=\mathbf{4 . 1 0 2}$ & IBI (India) & $\mathbf{4 . 2 6 0}$ \\
& JIF & $=\mathbf{1 . 5 0 0}$ & SJIF (Morocco) & $=\mathbf{2 . 0 3 1}$ & & \\
\hline
\end{tabular}

где коэффициенты $a_{0}, a_{1}, \ldots, a_{n}$-постоянные вещественные числа.

Для нахождения общего решения уравнения (1) воспользуемся стандартными средствами пакета современной компьютерной математики Maple. B Maple работа с дифференциальными уравнениями начинается с подключения специализированного пакета DEtools .

Найдем общее решение линейного дифференциального уравнения с постоянными коэффициентами 3-го порядка:

$$
y^{\prime \prime \prime}-6 y^{\prime \prime}+11 y^{\prime}-6 y=0
$$

Подключив пакет DEtools, вводим уравнение. Для производных функции при записи дифференциального уравнения используем команду прямого исполнения $\operatorname{diff}(f, x)$, первый аргумент которой есть дифференцируемая функция, а второй переменная, по которой надо брать производную. Для производных высших порядков указываем в параметрах $x \$ n$, где $n$ - порядок производной, т.е. $\operatorname{diff}(f, x \$ n)$ :

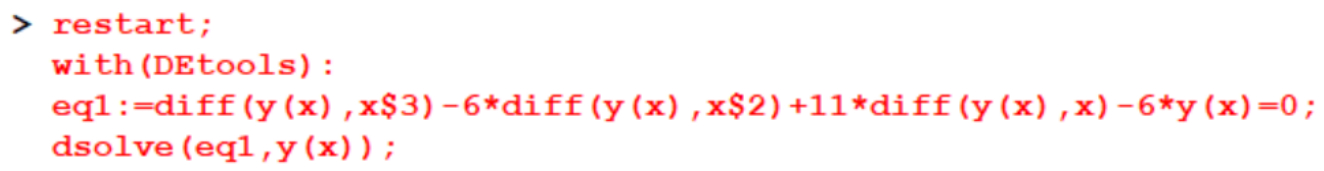

$$
\begin{gathered}
e q l=\left(\frac{\partial^{3}}{\partial x^{3}} \mathrm{y}(x)\right)-6\left(\frac{\partial^{2}}{\partial x^{2}} \mathrm{y}(x)\right)+11\left(\frac{\partial}{\partial x} \mathrm{y}(x)\right)-6 \mathrm{y}(x)=0 \\
\mathrm{y}(x)={ }_{-} C l \mathrm{e}^{x}+{ }_{-} C 2 \mathrm{e}^{(2 x)}+{ }_{-} C 3 \mathrm{e}^{(3 x)}
\end{gathered}
$$

Для нахождения аналитического решения данного дифференциального уравнений применяем команду dsolve (eq, var), где eq дифференциальное уравнение, var - неизвестная функция и получим общее решение указанного уравнения, в котором число постоянных равно порядку уравнения и имеют обозначения в системе Maple в виде _C1, $C 2 \ldots$.
Рассмотрим решение линейного дифференциального уравнения более высокого порядка, например, 5 -го порядка:

$$
y^{V}-2 y^{I V}+2 y^{I I I}-4 y^{I I}+y^{I}-2 y=0
$$

и применим выше описанный алгоритм:

$>$ restart

wuth (DEtools) :

eq2 : $=\operatorname{diff}(y(x), x \$ 5)-2 * \operatorname{diff}(y(x), x \$ 4)+2 * \operatorname{diff}(y(x), x \$ 3)-4 * \operatorname{diff}(y(x), x \$ 2)+\operatorname{diff}(y(x), x)-2 * y(x)=0$ dsolve (eq2, y (x)) ;

$$
\begin{gathered}
e q 2=\left(\frac{\partial^{5}}{\partial x^{5}} \mathrm{y}(x)\right)-2\left(\frac{\partial^{4}}{\partial x^{4}} \mathrm{y}(x)\right)+2\left(\frac{\partial^{3}}{\partial x^{3}} \mathrm{y}(x)\right)-4 \% 1+\left(\frac{\partial}{\partial x} \mathrm{y}(x)\right)-2 \mathrm{y}(x)=0 \\
\% 1=\frac{\partial^{2}}{\partial x^{2}} \mathrm{y}(x) \\
\mathrm{y}(x)={ }_{-} C 1 \cos (x)+{ }_{-} C 2 \sin (x)+{ }_{-} C 3 \mathbf{e}^{(2 x)}+{ }_{-} C 4 \sin (x) x+\ldots C 5 \cos (x) x
\end{gathered}
$$

Как видим, линейное дифференциальное однородное уравнение 3-го порядка и выше решается с помощью команды dsolve и не сопряжено с какими-либо затруднениями. Это дает возможность составить автоматизированную программу для решения линейного дифференциального уравнения 3-го порядка, которую можно в дальнейшем применять для нахождения общего решения линейных дифференциальных неоднородных уравнений более высших порядков вводя, лишь коэффициенты уравнения. 


\begin{tabular}{|c|c|c|c|c|c|c|}
\hline Impact Factor: & $\begin{array}{l}\text { ISRA (India) } \\
\text { ISI (Dubai, UAE } \\
\text { GIF (Australia) } \\
\text { JIF }\end{array}$ & $\begin{array}{r}=1.344 \\
=0.829 \\
=0.564 \\
=1.500\end{array}$ & $\begin{array}{l}\text { SIS (USA) } \\
\text { PИHЦ (Russia) } \\
\text { ESJI (KZ) } \\
\text { SJIF (Morocco) }\end{array}$ & $\begin{array}{l}=0.912 \\
=0.207 \\
=4.102 \\
=\mathbf{2 . 0 3 1}\end{array}$ & $\begin{array}{l}\text { ICV (Poland) } \\
\text { PIF (India) } \\
\text { IBI (India) }\end{array}$ & $\begin{array}{l}=6.630 \\
=1.940 \\
=4.260\end{array}$ \\
\hline
\end{tabular}

Запишем коэффициенты линейного дифференциального однородного уравнения 3 го порядка, которое имеет вид:

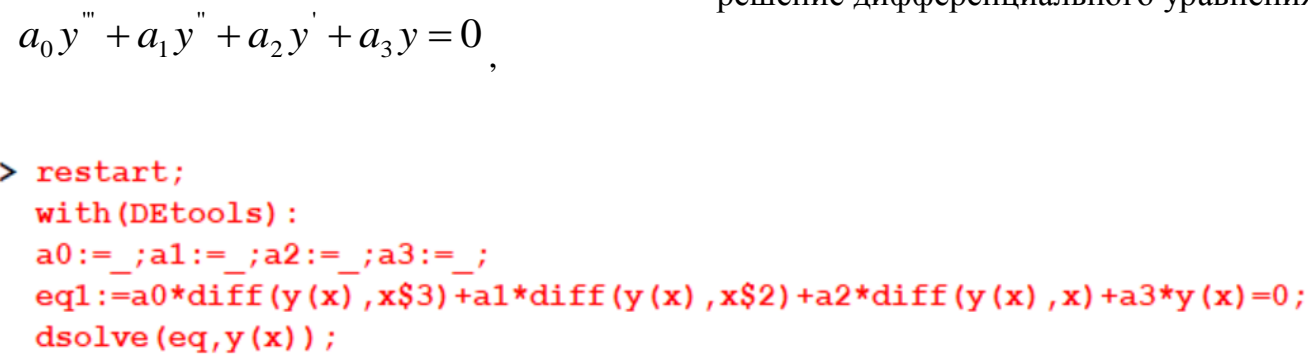

Рассмотрим нахождение общего решения линейного дифференциального неоднородного уравнения $n$-го порядка:

$$
a_{0} y^{(n)}+a_{1} y^{(n-1)}+a_{2} y^{(n-2)}+\ldots+a_{n-1} y^{\prime}+a_{n} y=f(x),
$$

где коэффициенты $a_{0}, a_{1}, \ldots, a_{n^{-}}$постоянные вещественные числа. Для решения уравнения (2) применяется метод подбора частного решения, если функция $f(x)$ имеет вид:

$$
f(x)=e^{\alpha x}(P(x) \cos \beta x+Q(x) \sin \beta x),
$$

или состоит из суммы такого рода функций. Во всех остальных случаях используют метод вариации произвольных постоянных. вводим дифференциальное уравнение и применяя команду dsolve(eq, var), получим общее решение дифференциального уравнения:
Найдем общее решение линейного дифференциального неоднородного уравнения 3го порядка:

$$
y^{\prime \prime}+3 y^{\prime \prime}-10 y^{\prime}=x-3,
$$

где $f(x)=x-3$ имеет вид (3), где $\alpha=0$, $\beta=0, P(x)=x-3$.

Применим тот же алгоритм решения средствами пакета Maple, как и при нахождении общего решения линейного дифференциального однородного уравнения:

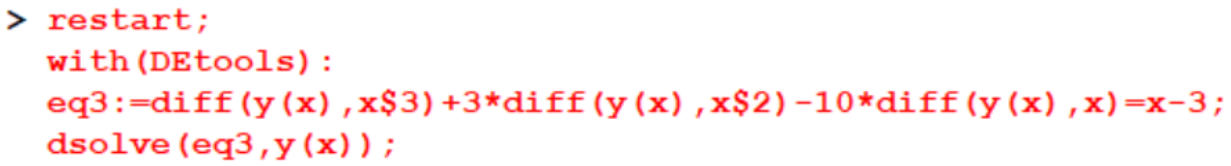

Как видим, общее решение линейного дифференциального неоднородного уравнения записано таким образом, что четко видна, структура этого решения - сумма общего решения соответствующего однородного дифференциального уравнения и частного решения этого же неоднородного дифференциального уравнения. В строке вывода, решение неоднородного линейного дифференциального уравнения состоит из слагаемых, которые содержат произвольные постоянные, что соответствует общему решению соответствующего однородного уравнения, и слагаемых без произвольных постоянных, что представляет частное решение этого же неоднородного дифференциального уравнения.

$$
\text { Рассмотрим решение линейного }
$$
дифференциального неоднородного уравнения 3го порядка следующего вида : 


\begin{tabular}{l|lr|ll|ll} 
& ISRA (India) & $=\mathbf{1 . 3 4 4}$ & SIS (USA) & $=\mathbf{0 . 9 1 2}$ & ICV (Poland) & $=\mathbf{6 . 6 3 0}$ \\
Impact Factor: & ISI (Dubai, UAE) $=\mathbf{0 . 8 2 9}$ & PUHЦ (Russia) $=\mathbf{0 . 2 0 7}$ & PIF (India) & $=\mathbf{1 . 9 4 0}$ \\
& GIF (Australia) & $\mathbf{0 . 5 6 4}$ & ESJI (KZ) & $=4.102$ & IBI (India) & $=\mathbf{4 . 2 6 0}$ \\
& JIF & $=\mathbf{1 . 5 0 0}$ & SJIF (Morocco) & $=2.031$ & & \\
\hline
\end{tabular}

$$
y^{\prime \prime \prime}-y^{\prime}=\sin 3 x
$$

и применим ранее описанный алгоритм нахождения общего решения линейного дифференциального однородного уравнения:

restart:

with (DEtools):

eq4 $:=\operatorname{diff}(y(x), x \$ 3)-\operatorname{diff}(y(x), x)=\sin \left(3^{*} x\right)$;

dsolve (eq4, y (x)) ;

$$
\begin{gathered}
e q 4:=\left(\frac{\partial^{3}}{\partial x^{3}} \mathrm{y}(x)\right)-\left(\frac{\partial}{\partial x} \mathrm{y}(x)\right)=\sin (3 x) \\
\mathrm{y}(x)=\frac{1}{30} \cos (3 x)+{ }_{-} C 1+{ }_{-} C 2 \mathbf{e}^{x}+{ }_{-} C 3 \mathbf{e}^{(-x)}
\end{gathered}
$$

Следовательно, для нахождения общего решения линейных дифференциальных неоднородных уравнений со специальной правой частью вида $f(x)=e^{\alpha x}(P(x) \cos \beta x+Q(x) \sin \beta x)$

используется тот же алгоритм, как и для линейных дифференциальных однородных уравнений.

Рассмотрим нахождение общего решения линейного дифференциального неоднородного уравнения, где функция $f(x)$ не является выражением вида (3). Данное уравнение решается с помощью метода вариации произвольных постоянных (метод Лагранжа).

Найдем общее решение линейного дифференциального неоднородного уравнения 3го порядка:

$$
y^{\prime \prime \prime}+y^{\prime}=\frac{1}{\cos x},
$$

используем алгоритм нахождения общего решения линейных дифференциальных неоднородных уравнений со специальной правой частью.

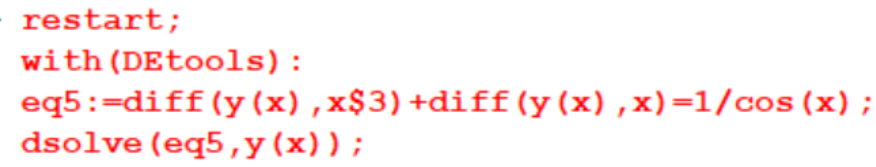

В записи общего решения слагаемые, соответствующие частному решению уравнения имеют сложные алгебраические выражения. Попробуем улучшить результат вычисления, используя команду dsolve, в параметрах которой укажем опцию output=basis.Данная опция дает возможность найти фундаментальную систему решений соответствующего однородного уравнения. Для нахождения решения используем функцию пакета varparam(sols,v,ivar), которая находит общее решение дифференциального уравнения sols методом вариации произвольных постоянных, $v$ задает правую часть уравнения, ivar задает переменную: 
$>$ restart;

with (DEtools):

eq5 : $=\operatorname{diff}(y(x), x \$ 3)+\operatorname{diff}(y(x), x)=1 / \cos (x)$;

eq6: $=\operatorname{diff}(y(x), x \$ 3)+\operatorname{diff}(y(x), x)=0$;

dsolve (eq6,y (x), output=basis) ;

sols : =dsolve (eq6,y (x), output=basis) ;

$\mathrm{y}:=\operatorname{varparam}(\operatorname{sols}, 1 / \cos (\mathrm{x}), \mathrm{x})$;

$$
\begin{gathered}
\text { eq } 5:=\left(\frac{\partial^{3}}{\partial x^{3}} \mathrm{y}(x)\right)+\left(\frac{\partial}{\partial x} \mathrm{y}(x)\right)=\frac{1}{\cos (x)} \\
e q 6=\left(\frac{\partial^{3}}{\partial x^{3}} \mathrm{y}(x)\right)+\left(\frac{\partial}{\partial x} \mathrm{y}(x)\right)=0 \\
{[1, \cos (x), \sin (x)]} \\
\operatorname{sols}:=[1, \cos (x), \sin (x)] \\
y=C_{1}+{ }_{-} C_{2} \cos (x)+C_{3} \sin (x)+\ln (\sec (x)+\tan (x))-x \cos (x)+\ln (\cos (x)) \sin (x)
\end{gathered}
$$

Сравнивая полученный результат вычисления общего решения уравнения с раннее имеющимся, видно, что выражение общего решения записано в более упрощенной форме. Теперь есть возможность составить общую программу решения уравнения

$$
y^{\prime \prime \prime}+y^{\prime}=\frac{1}{\cos x},
$$

которая содержит метод подбора частных решений и метод вариации произвольных постоянных, для нахождения общего решения любого линейного дифференциального неоднородного уравнения. Правую часть уравнения

$$
f(x)=e^{\alpha x}(P(x) \cos \beta x+Q(x) \sin \beta x)
$$
запишем в 2-х формах $f(x)=e^{\alpha x} P(x) \quad$ и $h(x)=e^{u x}(M(x) \cos v x+N(x) \sin v x), \quad$ при этом многочлены $P(x), Q(x), M(x), N(x)$ записываются с учетом степени присутствующего многочлена правой части. Правая часть, соответствующая методу вариации произвольных постоянных записывается в виде функции $g(x)$ :

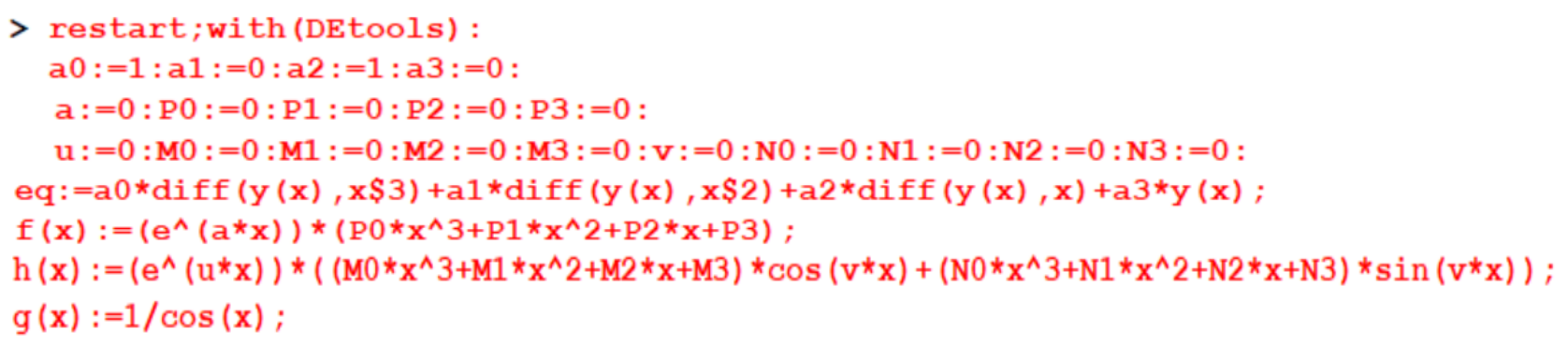

Зададим условие так, чтобы по виду правой части уравнения определила метод решения, получим:

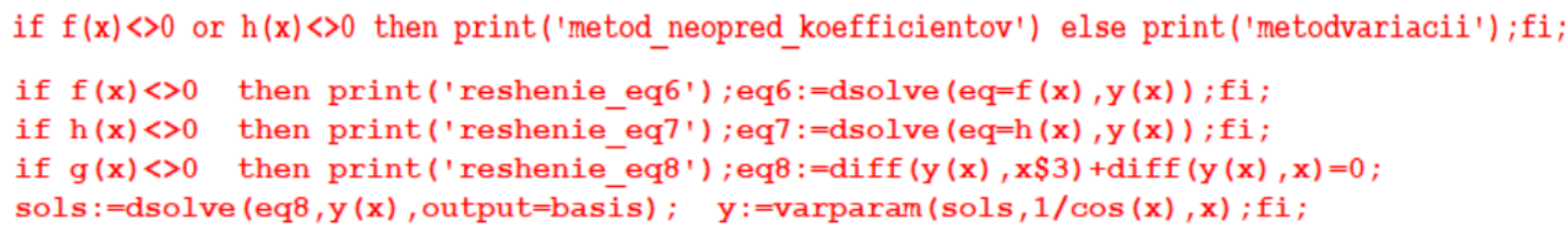




\begin{tabular}{l|lr|ll|ll} 
& ISRA (India) & $=\mathbf{1 . 3 4 4}$ & SIS (USA) & $=\mathbf{0 . 9 1 2}$ & ICV (Poland) & $=\mathbf{6 . 6 3 0}$ \\
Impact Factor: & ISI (Dubai, UAE) $=\mathbf{0 . 8 2 9}$ & PUHЦ (Russia) $=\mathbf{0 . 2 0 7}$ & PIF (India) & $=\mathbf{1 . 9 4 0}$ \\
& GIF (Australia) & $\mathbf{0 . 5 6 4}$ & ESJI (KZ) & $=\mathbf{4 . 1 0 2}$ & IBI (India) & $=\mathbf{4 . 2 6 0}$ \\
& JIF & $=\mathbf{1 . 5 0 0}$ & SJIF (Morocco) & $=\mathbf{2 . 0 3 1}$ & & \\
\hline
\end{tabular}

$$
\begin{gathered}
\left(\frac{\partial^{3}}{\partial x^{3}} \mathrm{y}(x)\right)+\left(\frac{\partial}{\partial x} \mathrm{y}(x)\right)=\frac{1}{\cos (x)} \\
\text { metodvariacii } \\
\text { reshenieeq } 8
\end{gathered}
$$

\section{Conclusion}

Таким образом, для использования программы нужно определить составляющие правой части дифференциального уравнения вида $f(x)=e^{\alpha x}(P(x) \cos \beta x+Q(x) \sin \beta x) \quad$ и ввести коэффициенты для одной из функций $f(x)=e^{\alpha x} P(x)$ или $h(x)=e^{u x}(M(x) \cos v x+N(x) \sin v x), \quad$ при этом $g(x)=0$. В противном случае коэффициенты приравниваются к нулю и записывается функция $g(x)$. Данную программу можно использовать для нахождения общего решения дифференциальных неоднородных уравнений любого порядка, вводя соответствующие коэффициенты уравнения.

\section{References:}

1. Erugin N.P et al. (1974) Kniga dlya chteniya po obshchemu kursu obyknovennykh uravneniy.M.,1974.,65-77

2. Kartashev E.A., Rozhdestvenskiy B.L. (1976) Obyknovennye differentsial'nye uravneniya i osnovy variatsionnogo ischisleniya. M.,1976.

3. Krasnov M.L., Makarenko G.I. (1978) Sbornik zadach po obyknovennym differentsial'nym uravneniyam.M.,1978.

4. N.M. Matveev. (1963) Metody integrirovaniya obyknovennykh differentsial'nykh.- Vysshaya shkola.,Moskva-1963.,336-467.

5. Petrovskiy I.G. (1970) Lektsii po teorii obyknovennykh differentsial'nykh uravneniyyu M.,1970.,154-200
6. (1965) Pod redaktsiey P.E. Debyuka., G.I. Kruchkovicha., Sbornik zadach po kursu vysshey matematiki.M.1965.

7. Pontryagin L.S. (1974) Obyknovennye differentsial'nye uravneniya. M,1974.,41-62

8. Stepanov V.V. (1959) Kurs differentsial'nykh uravneniy, M., 1959., 214-241.

9. Filippov A.F. (1973) Sbornik zadach po obyknovennym differentsial'nym uravneniyam. M., 1973., 60-75.

10. Tikhonov A.N., Vasil'eva A.B., Sveshnikov A.G. (2018) Differentsial'nye uravneniya., 3345 . 


\begin{tabular}{l|lr|ll|ll} 
& ISRA (India) & $=\mathbf{1 . 3 4 4}$ & SIS (USA) & $=\mathbf{0 . 9 1 2}$ & ICV (Poland) & $=\mathbf{6 . 6 3 0}$ \\
Impact Factor: & ISI (Dubai, UAE) & $=\mathbf{0 . 8 2 9}$ & PVHL (Russia) & $=\mathbf{0 . 2 0 7}$ & PIF (India) & $=\mathbf{1 . 9 4 0}$ \\
& GIF (Australia) & $=\mathbf{0 . 5 6 4}$ & ESJI (KZ) & $=\mathbf{4 . 1 0 2}$ & IBI (India) & $=\mathbf{4 . 2 6 0}$ \\
& JIF & $=\mathbf{1 . 5 0 0}$ & SJIF (Morocco) & $\mathbf{2 . 0 3 1}$ & & \\
\hline
\end{tabular}

